\title{
Modelado y control no lineal de ácidos grasos volátiles en un biorreactor anaerobio de lecho fijo y flujo ascendente (UAFBR) ${ }^{*}$
}

\author{
Iván Ramírez ${ }^{* *}$ \\ Andrés Ramírez-Villarreal ${ }^{* * *}$ \\ Juan Ospina-Nieto ${ }^{* * *}$
}

Recibido: 2018/10/18 • Aceptado: 2019/07/08

https://doi.org/10.22395/rium.v19n36a3

\begin{abstract}
Resumen
En este trabajo se presenta una estrategia de regulación robusta de los ácidos grasos volátiles (AGV) en un proceso de digestión anaerobia. La estrategia propuesta es una ley de control por retroalimentación no lineal obtenida por linealización entrada-salida que permite alcanzar el objetivo de control a pesar de errores de modelado (incertidumbres en la cinética de las reacciones) y las restricciones del actuador. Los experimentos fueron conducidos con un reactor anaerobio piloto de lecho fijo y flujo ascendente (UAFBR) con volumen activo de $0,947 \mathrm{~m}^{3}$, completamente equipado, utilizado para el tratamiento de aguas residuales provenientes de una destilería de vino en Narbonne, Francia. Con el objetivo de validar los modelos utilizados, resultados simulados y experimentales, son presentados y discutidos los parámetros de desempeño del controlador bajo los modelos AM2 y ADM1 (tiempo de subida, tiempo de establecimiento, sobre impulso y error en estado estacionario) obtenidos de las simulaciones numéricas, que prueban la eficiencia y aplicabilidad de la estrategia de control propuesta.
\end{abstract}

Palabras clave: ácidos grasos volátiles (AGV); biogás; biorreactor; demanda química de oxígeno (DQO); digestión anaeróbica (DA); control no lineal; modelo de digestión.

\footnotetext{
* Artículo producto de la fase en curso de modelado y control de un biorreactor anaerobio piloto del trabajo de grado titulado Control de un biorreactor anaeróbico de laboratorio financiado por la Universidad del Quindío.

** Doctor y magíster en Ingeniería, Profesor asistente, Facultad de Ingeniería, Universidad del Quindío, Colombia. Correo electrónico: idramirez@uniquindio.edu.co. Orcid: http://orcid.org/0000-0002-5660-8299

*** Estudiante de Ingeniería Electrónica, Facultad de Ingeniería, Universidad del Quindío, Colombia. Correo electrónico: aramiezv@uqvirtual.edu.co.Orcid: http://orcid.org/0000-0002-7407-7495

***** Estudiante de ingeniería electrónica, Facultad de Ingeniería, Universidad del Quindío, Colombia. Correo electrónico: jdospinan@uqvirtual.edu.co. Orcid: http://orcid.org/0000-0002-9000-1490
} 


\title{
Modeling and nonlinear control of volatile fatty acids in an upflow anaerobic fixed bed biofilm reactor (UAFBR)
}

\begin{abstract}
In this article a robust regulation strategy of volatile fatty acids is proposed by an anaerobic digestion process. The proposed strategy is a control law via nonlinear feedback obtained by input-output linearization that allows the completion of the control goals despite the modeling errors (uncertainties in the kinetics of the reactions) and the actuator restrictions. The experiments were conducted with an pilot fixed anaerobic reactor and ascending flow (UAFBR) with an active volume of $0.947 \mathrm{~m} 3$ fully equipped, used for the treatment of residual waters coming from a wine distillery in Narbonne, France. With the goal of validating the models used, simulated and experimental results are presented and discussed. The performance parameters of the controller under the AM2 and ADM1 models (up time, establishment time, over-impulse and stationary state error) obtained from the numeric simulations that prove the efficiency and applicability of the proposed control strategy.
\end{abstract}

Keywords: volatile fatty acids (AGV); bio-gas; bioreactor; chemical oxygen demand; anaerobic digestion; nonlinear control; digesting model.

\section{Modelagem e controle não linear de ácidos graxos voláteis em um biorreator anaeróbio de leito fixo e fluxo fixo ascendente}

\begin{abstract}
Resumo
Neste trabalho, apresenta-se uma estratégia de regulação robusta dos ácidos graxos voláteis (AGV) em um processo de digestão anaeróbia. A estratégia proposta é uma lei de controle por retroalimentação não linear obtida por linearização entrada-saída que permite atingir o objetivo de controle apesar de erros de modelagem (incertezas na cinética das reações) e das restrições do acionador. As experiências foram conduzidas com um reator anaeróbio-piloto de leito fixo e fluxo ascendente (UAFBR) com volume ativo de $0,947 \mathrm{~m}^{3}$, completamente equipado, utilizado para tratar águas residuais provenientes de uma destilaria de vinho em Narbonne, França. Com o objetivo de validar os modelos utilizados, resultados simulados e experimentais, são apresentados e discutidos os parâmetros de desempenho do controlador sob os modelos AM2 e ADM1 (tempo de subida, tempo de estabelecimento, sobre impulso e erro de estado estacionário) obtidos das simulações numéricas, que provam a eficiência e a aplicabilidade da estratégia de controle proposta.
\end{abstract}

Palavras-chave: ácidos graxos voláteis (AGV); biogás; biorreator; demanda química de oxigênio (DQO); digestão anaeróbica (DA); controle não linear; modelo de digestão. 


\section{INTRODUCCIÓN}

El desarrollo tecnológico acelerado ha causado escasez de combustibles fósiles, por ello en la actualidad se buscan estrategias que permitan reducir la inminente crisis energética. Los procesos anaerobios, como herramientas de solución al problema, contribuyen entre otras cosas a la generación de energías renovables. El proceso de digestión anaeróbica (DA) se ha usado con éxito en sectores industriales como el farmacéutico, el alimenticio y otros; sin embargo, en las últimas décadas surgen procesos ampliamente usados como el procesamiento de desechos orgánicos y el tratamiento de aguas residuales.

La digestión anaerobia es un proceso biológico multipaso en la que el carbón orgánico es convertido en sus estados más oxidados (i. e. dióxido de carbono $\mathrm{CO}_{2}$ ) y más reducidos (i. e. metano $\mathrm{CH}_{4}$ ). El principal producto es en efecto el biogás, una mezcla de $\mathrm{CH}_{4}$ y $\mathrm{CO}_{2}$ así como trazas de gases como sulfuro de hidrógeno $\left(\mathrm{H}_{2} \mathrm{~S}\right)$ e hidrógeno $\left(\mathrm{H}_{2}\right)$ [1]. El proceso se obtiene como resultado de una descomposición bioquímica consecutiva de polímeros en metano y dióxido de carbono en un ambiente donde una variedad de microorganismos crece armoniosamente ofreciendo sus productos más reducidos. Esos microorganismos incluyen bacterias fermentativas (acidogénicas), productoras de hidrógeno, bacterias productoras de acetato (acetogénicas) y arqueas productoras de metano (metanogénicas). Estas bacterias trabajan sintróficamente, ya que están ligadas fisiológica, cinética y termodinámicamente [2].

En comparación con los procesos de digestión aeróbica, la DA posee ventajas interesantes como [3]: (i) producción de energía renovable a partir de $\mathrm{CH}_{4}$ el cual puede utilizarse después para la recuperación de energía o incluso para producción de electricidad como en las celdas de combustible [4]; (ii) buen desempeño en la degradación de materiales orgánicos complejos; (iii) baja producción de lodos y (iv) reducción de agentes patógenos. Por otro lado, presenta desventajas como [5]: (i) microrganismos con tasas de crecimiento lento y dinámico que aún no son bien conocidas; (ii) alta sensibilidad a sobrecargas o perturbaciones que pueden conducir a inestabilidad del reactor.

Entre los principales retos que ofrece el control de la DA están la presencia de organismos vivos con reacciones metabólicas de alta complejidad (altamente no lineales y algunas veces impredecibles) y los costos prohibitivos de la instrumentación necesaria para el monitoreo de las variables de interés.

Los digestores anaerobios a menudo presentan importantes problemas de estabilidad que solo pueden evitarse a través del uso de estrategias de control apropiadas. Tales estrategias, en general, requieren el desarrollo de modelos matemáticos y la identificación de parámetros, estas tareas han sido un área activa de investigación durante los últimos tiempos, pues su complejidad es de gran interés en los ámbitos 
académicos y técnicos, e involucran diferentes disciplinas de la ciencia y la ingeniería en cuanto al diseño de estrategias de control se refiere. Este trabajo usa dos modelos: el modelo de la IWA de la digestión anaerobia ADM1 [6] y el modelo desarrollado por Bernard et al. [7], que subsiguientemente será denominado AM2.

El grupo de trabajo de la Asociación Internacional del Agua (IWA) para la modelización matemática del proceso de digestión anaeróbica desarrolló una modelo común: ADM1 que puede ser utilizado tanto por investigadores como técnicos [8]. El diseño estructurado del modelo ADM1 lo hace adecuado ser aplicación en diferentes situaciones encontradas en la práctica.

El ADM1 es un modelo estructurado que refleja los principales procesos involucrados en la conversión de sustratos orgánicos complejos en $\mathrm{CH}_{4}, \mathrm{CO}_{2}$ y subproductos inertes. ADM1 es una herramienta que permite predicciones con suficiente precisión para ser útil, sin embargo, debido a las demandas variables de operación y optimización de proceso, se requiere un grado diferente de calibración y validación del modelo en cada proceso.

En ADM1, tanto los procesos bioquímicos como los fisicoquímicos son incluidos. Todos los componentes excepto los inorgánicos se expresan en términos de su DQO. Las especies nitrogenadas y las especies con carbono inorgánico se describen en términos de sus concentraciones molares. Las rutas de reacción bioquímica incluyen: (i) una etapa de desintegración extracelular, que convierte material compuesto en carbohidratos, lípidos y proteínas, (ii) una etapa de hidrólisis enzimática extracelular que convierte los productos de desintegración en monosacáridos (MS), ácidos grasos de cadena larga (LCFA) y aminoácidos (AA), (iii) acidogénesis o fermentación de los productos hidrolíticos en hidrógeno, acetato y ácidos grasos volátiles (AGV), es decir, propionato, butirato y valerato, (iv) acetogénesis de AGV en acetato, (v) metanogénesis acetoclástica e hidrogenotrófica. Además, se tiene en cuenta el decaimiento de la biomasa.

Para describir estos mecanismos, el modelo emplea 26 variables de estado para describir el comportamiento de componentes solubles (representados con $S$ ) y componentes particulados (representados con $X$ ). Así mismo, el modelo aborda el carbono inorgánico $\left(\mathrm{CO}_{2}\right.$ y NaHCO 3 y especies nitrogenadas $\left(\mathrm{NH}_{3}\right.$ y NH $\left.\mathrm{NH}^{4+}\right)$. Todas las especies que se disocian en función del $\mathrm{pH}\left(\mathrm{AGV}\right.$ y $\left.\mathrm{NH}_{3}\right)$ tienen variables definidas tanto para las especies protonadas y no protonadas [2]. El modelo mantiene un equilibrio de carga entre las especies iónicas y, por lo tanto, hay variables para los aniones y cationes inorgánicos, incluido el ion hidrógeno. El modelo resuelve la concentración de iones de hidrógeno y el pH al garantizar la neutralidad química en la solución.

La selección del número de poblaciones bacterianas involucradas en el proceso DA está directamente relacionada a la complejidad del modelo. Dado que uno de los 
objetivos es obtener un modelo capaz de representar el fenómeno de desestabilización sosteniendo su simplicidad para el diseño de estrategias de control, Bernard et al. [7], desarrollaron uno en el cual las poblaciones de microorganismos pueden ser divididas en dos grupos de características homogéneas y la DA puede ser descrita por un proceso de dos pasos. En el primero (acidogénesis), las bacterias acidogénicas $\left(X_{1}\right)$ consumen el sustrato orgánico $\left(S_{1}\right)$ y producen $\mathrm{CO}_{2}$ y AGV $\left(S_{2}\right)$. La población de arqueas metanogénicas $\left(X_{2}\right)$ usa en un segundo paso los AGV como sustrato para crecimiento y produce $\mathrm{CO}_{2} \mathrm{y} \mathrm{CH}_{4}$. El modelo también incorpora equilibrios electroquímicos, para obtener la alcalinidad que juega un papel central en el monitoreo y control de los procesos de DA.

Acorde con Bernard et al. [7], las reacciones biológicas están descritas por (1).

Acidogénesis (con tasa de reacción $r_{1}=\mu_{1} X_{1}$ ).

$$
k_{1} S_{1} \rightarrow X_{1}+k_{2} S_{2}+k_{4} C O_{2}
$$

Metanogénesis (con tasa de reacción $r_{2}=\mu_{2} X_{2}$ )

$$
k_{3} \mathrm{~S}_{2} \rightarrow \mathrm{X}_{2}+k_{5} \mathrm{CO}_{2}+k_{6} \mathrm{CH}_{4}
$$

En donde $S_{1}$ : representa la concentración del sustrato orgánico caracterizado por la DQO (g/L), y $S_{2}$ : representa la concentración de AGV (mmol/L).

En los desarrollos matemáticos se asumió que $S_{2}$ compuesto principalmente de acetato, propionato y butirato, se comporta como acetato puro. Es importante anotar que la DQO total está compuesta de $S_{1}+S_{2} \cdot \mu_{1}$ y $\mu_{2}\left(\mathrm{~d}^{-1}\right)$, representando las tasas específicas de crecimiento de los microorganismos acidogénicos y metanogénicos respectivamente.

Para direccionar estos mecanismos, el modelo emplea seis variables de estado para describir el comportamiento de componentes solubles $\left(S_{1}, S_{2}\right)$ y componentes particulados $\left(X_{1}, X_{2}\right)$. La alcalinidad total definida como la suma de los ácidos disociados en el medio es representada con $Z$, y $C$ representa el carbono inorgánico total, compuesto de bicarbonato y $\mathrm{CO}_{2}$ disuelto.

Si denotamos por $\xi=\left[X_{1}, X_{2}, Z, S_{1}, S_{2}, C\right]^{T}$ el vector de variables de estado, el modelo escrito en forma matricial está dado por (2).

$$
\frac{d \xi}{d t}=K r(\xi)-D \xi-Q+F
$$

Las matrices $K, D, Q$ y $F$ pueden ser encontradas en [7]. 
A pesar de las ventajas ya mencionadas, la aplicación de los procesos de DA es frecuentemente limitada porque son muy sensibles a perturbaciones y se tornan inestables. Tal inestabilidad se observa por la caída en la tasa de producción de metano, en $\mathrm{pH}$ o por una acumulación en la concentración de los AGV, lo que conduce a la falla del biodigestor. Esto hace deseable un control fino de los AGV que garantice la estabilidad del proceso [9].

Han sido publicados múltiples estudios en el diseño de estrategias de control de digestores anaerobios. A manera de ejemplo, Selesteanu et al. [10] utilizaron control adaptativo y control inteligente para estabilizar la biomasa al interior de los biorreactores utilizados, un tanque de reacción continuamente agitado (CSTR) y un biorreactor de alimentación por lotes, considerando las no linealidades y las características no estacionarias de los procesos, sin embargo, las estrategias de control propuestas no tienen la suficiente robustez, dado que se derivan de valores estimados de los parámetros cinéticos o de asumir la disponibilidad de las variables claves. Kravaris y Savoglidis [11] formularon una estrategia por modos deslizantes con el objetivo de encontrar la tasa de dilución óptima que permitió maximizar la producción de $\mathrm{CH}_{4}$ en un biorreactor continuo. El método de control por modos deslizantes fue seleccionado, tanto por su sólida fundamentación teórica como por sus ventajas prácticas, que incluyen, robustez y rápida respuesta. Khan y Loh [12] desarrollaron estrategias de linealización entrada-salida y backstepping para regular la concentración de biomasa en un CSTR, los resultados obtenidos fueron satisfactorios, demostrando la eficiencia de dichos enfoques. Las estrategias de control discutidas a pesar de sus buenos resultados, han sido diseñadas en simulaciones a partir de modelos dinámicos que consideran una sola población bacteriana.

El diseño de controladores de DA a partir de modelos con una y dos poblaciones microbianas generan incertidumbres en su implementación en las plantas de tratamiento de aguas residuales (PTAR), ya que estos no consideran la presencia de la diversidad microbiana al interior del biodigestor [13]. Sin embargo, los modelos que usan varias poblaciones microbianas a pesar de ser más realísticos, poseen demasiados estados y los hacen poco recomendables para el diseño de estrategias de control.

Ahora bien, como lo revelan los párrafos anteriores, muchos estudios se han dedicado a la estabilidad de los procesos de la DA a través del control de variables como concentración de biomasa, tasa de producción de metano, $\mathrm{pH}$ y demanda química de oxígeno (DQO), sin embargo, se han reportado pocos estudios sobre el control de los AGV. En este trabajo, los modelos ADM1 y AM2 se validan mediante resultados simulados con Matlab ${ }^{\mathrm{TM}} /$ Simulink y datos experimentales obtenidos de un reactor anaerobio de lecho fijo y flujo ascendente (UAFBR) usado para el tratamiento de aguas residuales provenientes de una destilería de vino en Narbonne-Francia. Los modelos se 
utilizan con el objetivo de establecer una estrategia de regulación robusta por retroalimentación lineal de entrada-salida de los ácidos grasos volátiles (AGV). Debido a la diferencia dimensional de los modelos empleados, se presenta una novedosa interfaz de asociación de variables, que permita realizar la simulación del controlador diseñado a partir del modelo AM2 sobre el modelo ADM1. Los parámetros de desempeño del controlador obtenidos de las simulaciones numéricas, sirven para probar la eficiencia y la aplicabilidad de la estrategia de control propuesta.

Este documento se encuentra organizado de la siguiente manera: en la sección 1 se da una descripción del reactor (UAFBR) utilizado y de su instrumentación, se plantean los modelos dinámicos empleados y se muestra el desarrollo de la interfaz entre ADM1 y AM2. Los resultados obtenidos del modelado y control con ADM1 se presentan en la sección 2. Finalmente, en la sección 3, se discuten y concluye sobre los resultados obtenidos.

\section{MATERIALES Y MÉTODOS}

\subsection{Montaje experimental}

El diagrama esquemático del biorreactor piloto UAFBR usado en este estudio es mostrado en la figura 1 . El reactor es circular con capacidad de $0,948 \mathrm{~m}^{3}$, cuenta con un sistema de dilución de 200L y un intercambiador de calor para mantener el sustrato a la temperatura deseada. El área de la superficie de soporte es de $135 \mathrm{~m}^{2}$ (Cloisonyl: $\left.180 \mathrm{~m}^{2} / \mathrm{m}^{3}\right)$. El reactor está instrumentado con sensores on line para la medición de $\mathrm{pH}$, temperatura, presión, flujo y concentración de gas, concentración AGV y DQO. El sustrato usado para la alimentación es agua residual de las destilerías de vino industrial, provenientes de bodegas locales ubicadas en el sector de Narbonne, Francia. Más detalles sobre el proceso se pueden encontrar en [14].

\subsection{AM2}

El modelo dinámico AM2, considerando solo cuatro de los seis estados, propuesto por Bernard et al. [7], está dado por (3).

$$
\begin{gathered}
\dot{x}_{1}=\left(\alpha_{\mathrm{f}}\left(x_{3}\right)-a D\right) x_{1} \\
\dot{x}_{2}=\left(\mu_{2}\left(x_{4}\right)-a D\right) x_{2} \\
\dot{x}_{3}=\left(x_{3, \text { in }}-x_{3}\right) D-k_{1} \mu_{1}\left(x_{3}\right) x_{1} \\
\dot{x}_{4}=\left(x_{4, i n}-x_{4}\right) D+k_{2} \alpha_{1}\left(x_{3}\right) x_{1}-k_{3} \propto_{2}\left(x_{4}\right) x_{2}
\end{gathered}
$$




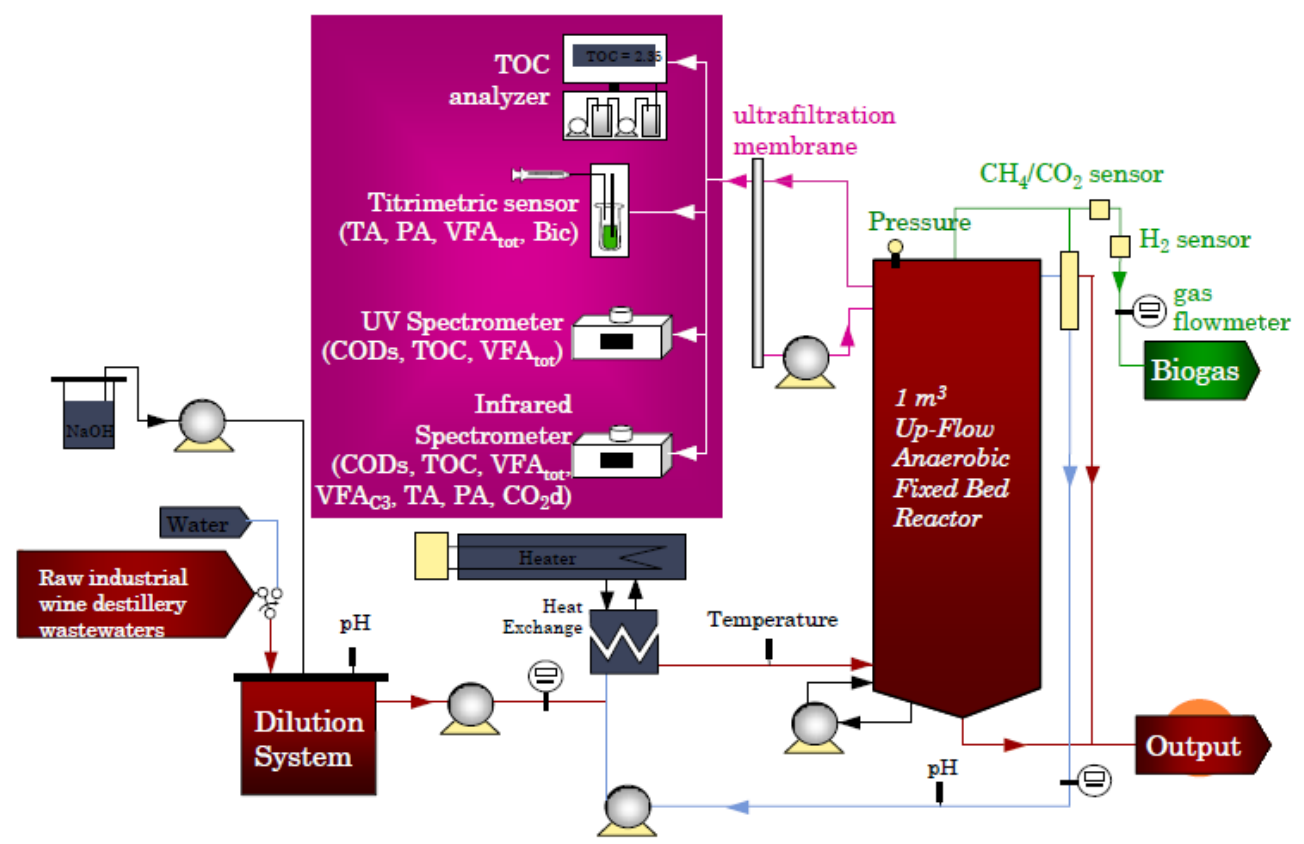

Figura 1. Vista esquemática del UAFBR y su instrumentación [14]

Fuente: elaboración propia

Donde: $x_{1}(\mathrm{~g} / \mathrm{L})$ es la concentración de las bacterias acidogénicas $\left(X_{1}\right) ; x_{2}(\mathrm{~g} / \mathrm{L})$ es la concentración de las arqueas metanogénicas $\left(X_{2}\right) ; x_{3}(\mathrm{~g} / \mathrm{L})$ es la concentración de DQO $\left(S_{1}\right) ; x_{4}(\mathrm{mmol} / \mathrm{L})$ es la concentración de $\operatorname{AGV}\left(S_{2}\right)$ y $D\left(\mathrm{~d}^{-1}\right)$ es la tasa de dilución ( $u$ desde el punto de vista de control).

Las tasas de crecimiento específico de las bacterias acidogénicas y metanogénicas están dadas por (4).

$$
\begin{gathered}
\mu_{1}\left(x_{3}\right)=\frac{\mu_{1, \max } x_{3}}{x_{3}+k_{s 1}} \\
\mu_{2}\left(x_{4}\right)=\frac{u_{2, \max } x_{4}}{x_{4}+k_{s 2}+\left(x_{4} / k_{I 2}\right)^{2}}
\end{gathered}
$$

En la tabla 1, se relacionan los diferentes parámetros utilizados en el modelo.

\subsection{ADM1}

Como se mencionó antes, el modelo usado para simulación es ADM1. Solo unos cuantos parámetros (principalmente $K_{m}$ y $K_{s}$ relacionados con los degradadores de 
acetato y propionato) fueron modificados para simular los datos experimentales del proceso (ver tabla 2). La diferencia entre el tiempo de retención hidráulica (HRT) y el tiempo de retención de sólidos (SRT) debido al biofilm presente en el reactor fue modelado adicionando un término extra $\left(t_{\text {res,X}}\right)$ en las ecuaciones de la biomasa, como recomienda el reporte de ADM1 [6].

Tabla 1. Parámetros utilizados en el modelo AM2 [7]

\begin{tabular}{clrc}
\hline Parámetro & \multicolumn{1}{c}{ Nombre } & Valor & Unidad \\
\hline$\alpha$ & Fracción de biomasa en el líquido & 0,50 & $\mathrm{mmol} / \mathrm{l}$ \\
\hline$k_{1}$ & Rendimiento de degradación de COD & 42,14 & - \\
\hline$k_{2}$ & Rendimiento de producción de VFA & 116,50 & $\mathrm{mmol} / \mathrm{g}$ \\
\hline$k_{3}$ & Rendimiento de consumo de VFA & 268,00 & $\mathrm{mmol} / \mathrm{g}$ \\
\hline$k_{1}$ & Constante de saturación media asociada a $x_{3}$ & 7,10 & $\mathrm{~g} / \mathrm{l}$ \\
\hline$k s_{2}$ & Constante de saturación media asociada a $x_{4}$ & 9,28 & $\mathrm{mmol} / \mathrm{l}$ \\
\hline$k l_{2}$ & Constante de inhibición asociada a $x_{4}$ & 256,00 & $\mathrm{mmol} / \mathrm{l}$ \\
\hline$\mu_{1, \max }$ & Tasa de crecimiento máximo para $x_{1}$ & 1,20 & $\mathrm{~d}-1$ \\
\hline$\mu_{2, \max }$ & Tasa de crecimiento máximo para $x_{2}$ & 0,74 & $\mathrm{~d}-1$ \\
\hline$x_{3, \text { in }}$ & composición a la entrada de $x_{3}$ & 25,00 & $\mathrm{~g} / \mathrm{l}$ \\
\hline$x_{4, \text { in }}$ & composición a la entrada de $x_{4}$ & 75,00 & $\mathrm{mmol} / \mathrm{l}$ \\
\hline & & &
\end{tabular}

Tabla 2. Parámetros estimados para ajuste de datos experimentales [15]

\begin{tabular}{ccc}
\hline Parámetro & Acetato & Propionato \\
\hline$K_{m D Q O} / \mathrm{kg}_{D Q O} \cdot$ dia $)$ & $1,93(8)^{*}$ & $2,51(13)$ \\
\hline$K_{s}\left(\mathrm{~kg}_{D Q O} / \mathrm{m}^{3}\right.$ & $1,41(0,15)$ & $1,41(0,10)$ \\
\hline
\end{tabular}

* Valores en paréntesis son los valores de referencia recomendados en ADM1

Fuente: elaboración propia

Las aguas residuales de las destilerías de vino usadas como influente durante los experimentos están compuestas de carbohidratos, azúcares, AGV, carbono inorgánico y nitrógeno. Las concentraciones de esos componentes individuales usados en ADM1 y AM2 como entradas del proceso se muestran en la tabla 3.

\subsection{Asociación entre variables de AM2 y ADM1}

A fin de usar los datos simulados con ADM1 en la validación del mismo y en la implementación numérica del controlador no lineal, proponemos un procedimiento 
de asociación de estados sistemático y genérico (interfaz) para obtener un modelo simplificado a partir de cualquier ADM1 validado.

Tabla 3. Concentración del influente del experimento [15]

\begin{tabular}{lcc}
\hline \multicolumn{1}{c}{ Parámetro } & Valor & Unidad \\
\hline Azúcares & 6,6439 & $\mathrm{Kg} \mathrm{COD} / \mathrm{m}^{3}$ \\
\hline Valerato & 0,5307 & $\mathrm{Kg} \mathrm{COD} / \mathrm{m}^{3}$ \\
\hline Butirato & 2,7015 & $\mathrm{Kg} \mathrm{COD} / \mathrm{m}^{3}$ \\
\hline Propionato & 1,6031 & $\mathrm{Kg} \mathrm{COD} / \mathrm{m}^{3}$ \\
\hline Acetato & 2,3279 & $\mathrm{Kg} \mathrm{COD} / \mathrm{m}^{3}$ \\
\hline Carbón inorgánico & 0,0025 & $\mathrm{Kmol} \mathrm{C} / \mathrm{m}^{3}$ \\
\hline Nitrógeno inorgánico & 0,0417 & $\mathrm{Kmol} \mathrm{N} / \mathrm{m}^{3}$ \\
\hline Carbohidratos & 1,1029 & $\mathrm{Kg} \mathrm{COD} / \mathrm{m}^{3}$ \\
\hline Degradadores de AGV & 20,0000 & $\mathrm{Kg} \mathrm{COD} / \mathrm{m}^{3}$ \\
\hline \multicolumn{2}{c}{ Fuente: elaboración propia } \\
\end{tabular}

Las diferentes especies de bacterias modeladas en ADM1 son agrupadas en AM2 en dos variables de estado. El primero, $X_{1}$, responsable de la acidogénesis, es la suma de las concentraciones de la biomasa de los microorganismos degradadores de azúcares, aminoácidos y ácidos grasos de cadena larga. Los microorganismos capaces de generar directamente $\mathrm{CH}_{4}$, es decir, los responsables de la metanogénesis, representan el segundo estado, $X_{2}$. La concentración de DQO ( $x_{3}$, en AM2), corresponde a la cantidad de materia orgánica degradable en ADM1 y finalmente, la suma de las concentraciones de los compuestos solubles que pueden ser convertidos en $\mathrm{CH}_{4}$ al final de la DA equivalen a la concentración de AGV ( $x_{4}$, en AM2). La interfaz de asociación descrita se muestra en la tabla 4.

Tabla 4. Interfaz de asociación entre los estados de AM2 y ADM1

\begin{tabular}{ccc}
\hline $\mathrm{AM} 2$ & $\mathrm{ADM} 1$ & Conversión \\
\hline$X_{1}[\mathrm{~g} / \mathrm{l}]$ & $X_{s s}, X_{a a}, X_{f a}\left[\mathrm{KgCOD} / \mathrm{m}^{3}\right]$ & $\mathrm{X}_{\mathrm{ss}}+\mathrm{X}_{\mathrm{aa}}+\mathrm{X}_{\mathrm{fa}}$ \\
\hline$X_{2}[\mathrm{~g} / \mathrm{l}]$ & $\mathrm{X}_{\mathrm{c},}, \mathrm{X}_{\mathrm{pro}}, \mathrm{X}_{\mathrm{ac}}, \mathrm{X}_{\mathrm{H}_{2}}\left[\mathrm{KgCOD} / \mathrm{m}^{3}\right]$ & $X_{c 4}+X_{p r o}+X_{a c}+X_{H_{2}}$ \\
\hline$S_{1}[\mathrm{~g} / \mathrm{l}]$ & $S_{s u}, S_{a a}, S_{f a}, S_{v a}, S_{b u}, S_{p r o}, S_{a c}, S_{H_{2}}, S_{C H_{4}}, S_{I}\left[\mathrm{KgCOD} / \mathrm{m}^{3}\right]$ & $S_{s u}+S_{a a}+S_{f a}+S_{v a}+S_{b u}+S_{p r o}+S_{a c}+S_{H_{2}}+S_{C H_{4}}+S_{I}$ \\
\hline$S_{2}[\mathrm{mmol} / \mathrm{l}]$ & $S_{v a}, S_{b u}, S_{p r o}, X_{a c}\left[\mathrm{KgCOD} / \mathrm{m}^{3}\right]$ & $\left(S_{v a}+S_{b u}+S_{p r o}+S_{a c}\right) * 1.000 / 64$ \\
\hline \multicolumn{2}{c}{ Fuente: elaboración propia }
\end{tabular}




\section{RESULTADOS}

\subsection{Modelos vs. datos experimentales}

Los datos experimentales después de un mes de monitoreo del UAFBR son mostrados en la figura 2 y figura 3, junto con las simulaciones de ADM1 y AM2.
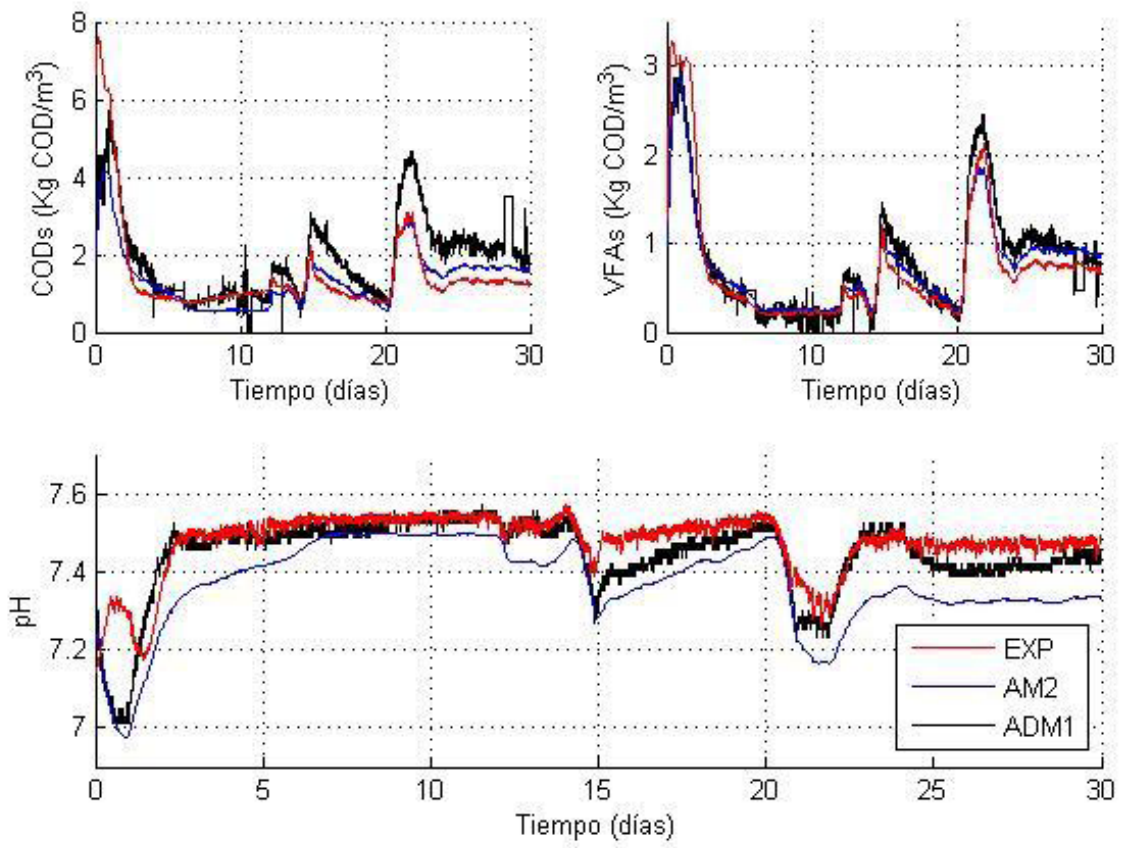

Figura 2. Datos experimentales (en negro) vs simulaciones de ADM1 (en rojo) y AM2 (en azul)

Fuente: elaboración propia

\subsection{Controlador no lineal}

Como estrategia de control no lineal se propone una linealización entrada-salida del sistema que regule la concentración de AGV, para esto se definen los vectores $f(x)$, $g(x)$ y $h(x)$ obtenidos del modelo del sistema (ecuación (3)), estos se presentan en (5).

$$
f(x)=\left[\mu_{1}\left(x_{3}\right) X_{1} \mu_{2}\left(x_{4}\right) x_{2}-k_{1} \mu\left(x_{3}\right) x 1 g(x)=\left[-a x 1-a x 2\left(x_{3, \text { in }} h(x)=x_{4}\right.\right.\right.
$$

El grado relativo $r$ según las condiciones dadas en (6), es $r=1$.

$$
\begin{gathered}
L g L^{j} f h(x)=0 ; j=0,1,2, \ldots, r-2 \\
L g L^{r-1} f h(x) \neq 0
\end{gathered}
$$




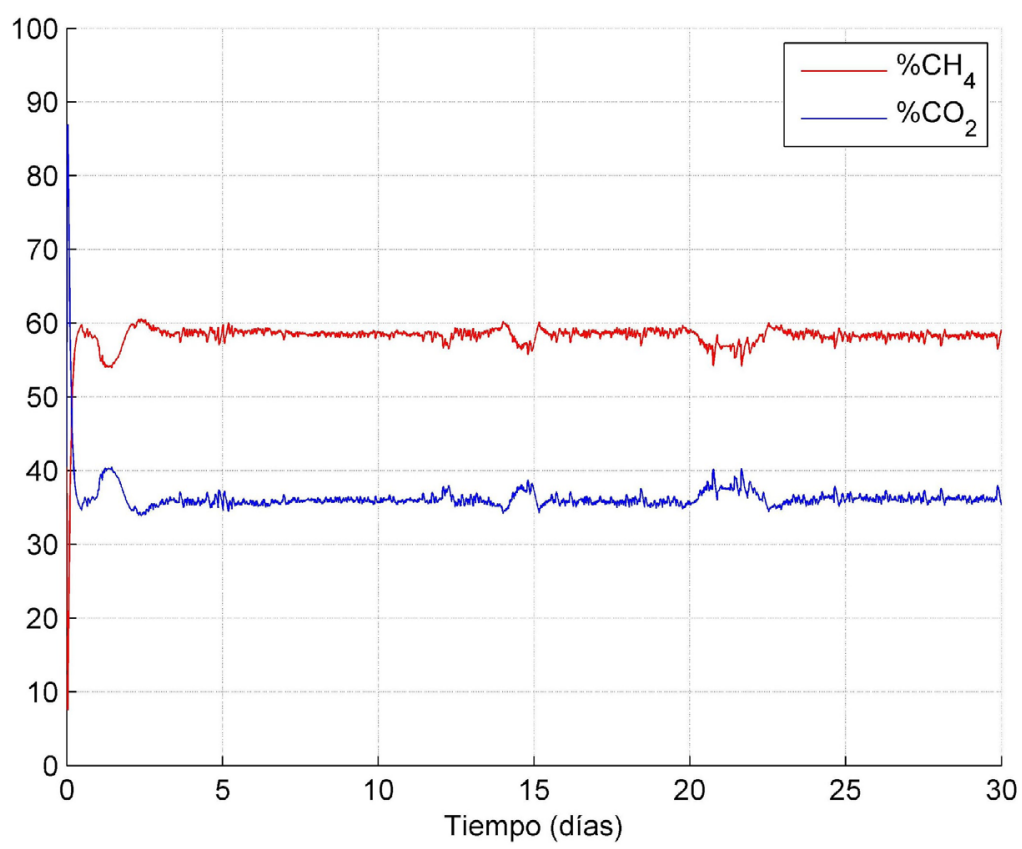

Figura 3. Simulación de la composición de biogás a partir de ADM1, porcentaje de $\mathrm{CH}_{4}$ (en rojo) y porcentaje de $\mathrm{CO}_{2}$ (en azul) Fuente: elaboración propia

Al existir un grado relativo bien definido, es posible encontrar un mapa invertible $z=\phi(x)$, que permita escribir el sistema en forma canónica normal. Se define como primera coordenada de la transformación difeomórfica $z_{1}=x_{4}$ y las $r-n$ coordenadas que completan el mapa invertible, deben satisfacer la ecuación $\operatorname{Lg} \phi_{r+j}(x)=o .(r=1, j=1, \ldots, n-r$.

La transformación difeomórfica propuesta está dada por (7). El mapeo es invertible dado que $\left[\frac{\delta \phi(x)}{\delta x}\right]$ es diferente de cero.

$$
\phi(x)=\left(x_{4} x_{2} / x_{1} x_{1} /\left(x_{3, \text { in }}-x_{3}\right)^{\alpha} x_{2} /\left(x_{4, \text { in }}-x_{4}\right)^{\alpha}\right)
$$

El sistema en forma canónica normal está representado en (8).

$$
z_{1}=\left(x_{4, \text { in }}-x_{4}\right) D+k_{2} \mu_{1}\left(x_{3}\right) x_{1}-k_{3} \mu_{2}\left(x_{4}\right) x_{2}
$$




$$
\begin{gathered}
\dot{\eta}_{1}=-\frac{\mu_{1}\left(x_{3}\right) x_{2}}{x_{1}}+\frac{\mu_{2}\left(x_{4}\right) x_{2}}{x_{1}} \\
\dot{\eta}_{2}=\frac{\mu_{1}\left(x_{3}\right) x_{1}}{\left(x_{3, \text { in }} \cdot x_{3}\right)^{\alpha}}-\frac{k_{1} \mu_{1}\left(x_{3}\right) x_{1}^{2} \alpha}{\left(x_{3, \text { in }}-x_{3}\right)^{\alpha+1}} \\
\dot{\eta}_{3}=\frac{\mu_{2}\left(x_{4}\right) x_{2}}{\left(x_{4, \text { in }}-x_{4}\right)^{\alpha}}+\frac{\alpha x_{2} k_{2} \mu_{1}\left(x_{3}\right) x_{1}}{\left(x_{4, \text { in }}-x_{4}\right)^{\alpha+1}}-\frac{\alpha k_{3} \mu_{2}\left(x_{4}\right) x_{2}^{2}}{\left(x_{4, \text { in }}-x_{4}\right)^{\alpha+1}}
\end{gathered}
$$

Una vez que $Z$ y sus $n-r$ derivadas se han hecho cero, la dinámica remanente, $\dot{\eta}(0, \eta)$, está dada por (9).

$$
\begin{gathered}
\dot{\eta}_{1}=-\frac{\mu_{1}\left(x_{3}\right) x_{2}}{x_{1}} \\
\dot{\eta}_{2}=-\frac{\mu_{1}\left(x_{3}\right) x_{1}}{\left(x_{3, \text { in }}-x_{3}\right)^{\alpha}}-\frac{k_{1} \mu_{1}\left(x_{3}\right) x_{1}^{2} \alpha}{\left(x_{3, \text { in }}-x_{3}\right)^{\alpha+1}} \\
\dot{\eta}_{3}=\frac{\alpha x_{2} k_{2} \mu_{1}\left(x_{3}\right) x_{1}}{\left(x_{4, \text { in }}\right)^{\alpha+1}}
\end{gathered}
$$

La dinámica remanente es asintóticamente estable si y solo si se cumple que la función candidata de Lyapunov (10) sea definida positiva y su derivada (11) semidefinida negativa.

$$
\begin{gathered}
v(x)=\frac{1}{2}\left(\eta_{1}^{2}+\eta_{2}^{2}+\eta_{2}^{3}\right) \\
\dot{v}(x)=\dot{\eta}_{1} \dot{\eta}_{1}+\dot{\eta}_{2} \dot{\eta}_{2}+\dot{\eta}_{3} \dot{\eta}_{3} \\
=-\frac{x_{2}}{x_{1}} \frac{\mu_{1}\left(x_{3}\right) x_{2}}{x_{1}}+\frac{x_{1}^{2} \mu_{1}\left(x_{3}\right)}{\left(x_{3, \text { in }}-x_{3}\right)^{2 \alpha+1}}\left[1-k_{1} x_{1} \alpha\left(x_{3, \text { in }}-x_{3}\right)^{-1}\right] \ldots \\
+\frac{\alpha x_{2}^{2} k_{2} \mu_{1}\left(x_{3}\right) x_{1}}{\left(x_{4, \text { in }}\right)^{2 \alpha+1}}
\end{gathered}
$$

La dinámica remante es estable, ya que las inecuaciones $\left.x_{3, \text { in }}-x_{3}\right)>0$, $k_{1} x_{1} \alpha\left(x_{3, \text { in }}-x_{3}\right)^{-1}>1$ y $\eta_{3} \dot{\eta}_{3}<\eta_{1} \dot{\eta}_{1}+\eta_{2} \dot{\eta}_{2}$, se sostienen en las condiciones de operación del reactor $\left(x_{1}, x_{2} \neq 0\right),\left(x_{1}, x_{2} \neq 0\right),\left(x_{i, i n}-x_{i}>0\right)$ y $\left(\mu_{1, \max }>\infty D^{*}\right)$.

El cálculo de la ley de control está dado por (12). 


$$
\mu=\frac{-L_{f}^{r} h(x)-m_{r-1} L_{f}^{r-1} h(x)-\cdots m_{1} L_{f} h(x)-m_{0} h(x)}{L_{g} L_{f}^{r-1} h(x)}
$$

Y finalmente la ley que regula los AGV es (13):

$$
\mu=\frac{-k_{2} \mu_{1}\left(x_{3}\right) x_{1}+k_{3} \mu_{2}\left(x_{4}\right) x_{2}-m_{0}\left(x_{4}-\underline{x}_{4}\right)}{x_{4, \text { in }}-x_{4}}
$$

Las respuestas simuladas de los AGV con una entrada multipaso, obtenidas usando la estrategia de control propuesta sobre ambos modelos ADM1 y AM2, se presentan en la figura 4 y los parámetros de desempeño del controlador se muestran en la tabla 5.

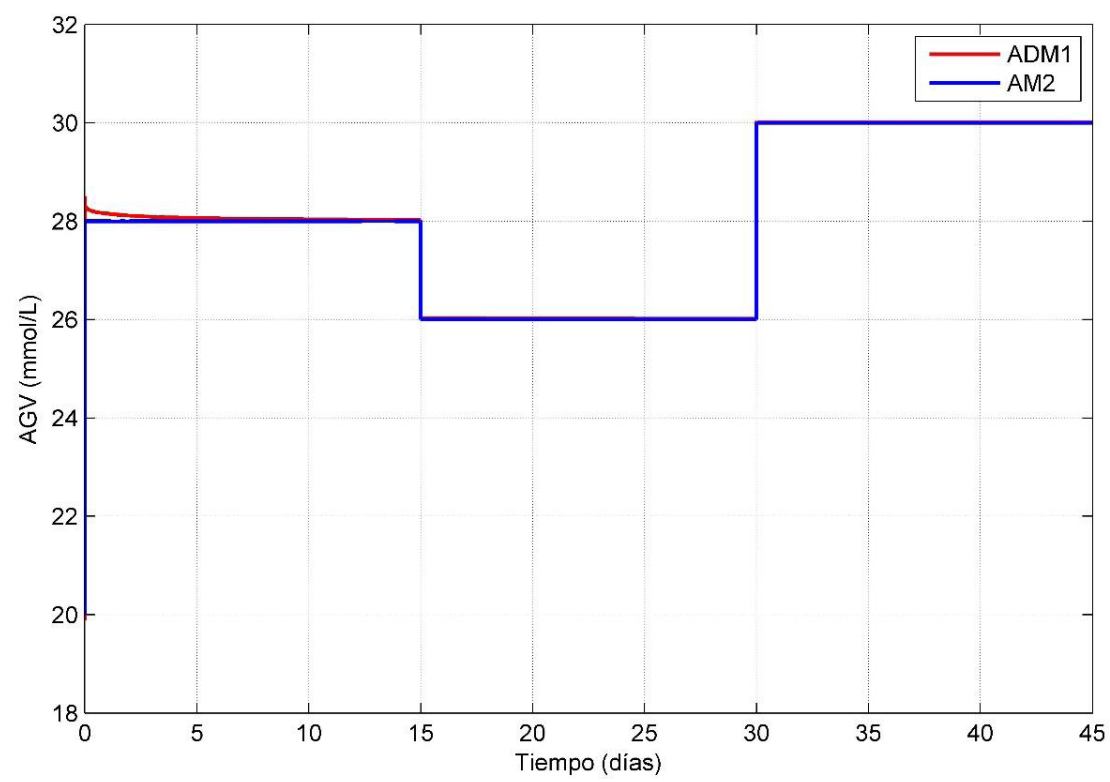

Figura 4. Comportamiento de la variable controlada ante cambios de referencia tipo escalón ( $28 \mathrm{mmol} / \mathrm{L}$ del día 0 al 15, $26 \mathrm{mmol} / \mathrm{L}$ del día 15 al 30, $30 \mathrm{mmol} / \mathrm{L}$ del día 30 al 45) Fuente: elaboración propia

Tabla 5. Parámetros de desempeño del controlador

\begin{tabular}{lcc}
\hline \multicolumn{1}{c}{ Parámetro } & ADM1 & AM2 \\
\hline$t_{r}(\min )(10-90 \%)$ & 0,72 & 1,44 \\
\hline$t_{s}(\min )$ & 3,6 & 2,16 \\
\hline
\end{tabular}




\begin{tabular}{lcc}
\hline$e_{s s}(\%)$ & 0,5 & $\sim 0$ \\
\hline O.S $(\%)$ & 0,1 & 0,006 \\
\hline \multicolumn{3}{c}{ Fuente: elaboración propia }
\end{tabular}

\section{DISCUSIÓN}

Como puede observarse, ambos modelos captan la evolución dinámica de las principales variables en la fase líquida, no obstante la fase gaseosa no es posible simularla desde AM2, ya que el modelo no contempla las presiones parciales del biogás.

Dado que ADM1 mantiene un equilibrio de carga entre las especies iónicas y contiene variables para los aniones y cationes inorgánicos, incluido el ion hidrógeno, y que el modelo resuelve la concentración de iones de hidrógeno y, por lo tanto, el $\mathrm{pH}$ al garantizar la neutralidad química en la solución, el pH es simulado con mayor precisión en ADM1. Ambos modelos subestiman las concentraciones de AGV. Parece ser que la velocidad a la cual el acetato se convirtió en metano bajo la carga impuesta fue algo sobreestimada en las simulaciones. Esto pudo deberse a la subestimación de los coeficientes de consumo de sustrato para la metanogénesis acetoclástica o a una sobreestimación de la inhibición de esta actividad por parte del amoniaco.

Como consecuencia, la evaluación de si un modelo es más apropiado que el otro es una tarea tediosa, más no imposible. Por supuesto, podría decirse que se pudo haber obtenido un mejor ajuste, pero el objetivo principal de este estudio no fue ajustar estos datos a la perfección sino evaluar la capacidad de ambos modelos para predecir adecuadamente el comportamiento de este proceso de digestión particular.

A pesar de que el diseño de la estrategia de control se hizo sobre AM2, los resultados de las simulaciones prueban que un buen desempeño del controlador (bajo tiempo de subida, sobre impulso despreciable y error de estado estacionario insignificante), puede ser logrado con la implementación de la estrategia de control propuesta en esta investigación en un modelo más complejo como ADM1, garantizando el buen desempeño del controlador en el UAFBR bajo investigación. Es de notar que los parámetros de desempeño del controlador sobre ambos modelos es prácticamente el mismo, puesto que están expresados en minutos, que es una unidad muy pequeña en cuanto a funcionamiento de reactores se trata. La estrategia de control está siendo implementada sobre el reactor piloto en Narbonne-Francia y los resultados serán publicados posteriormente.

\section{CONCLUSIONES}

Teniendo en cuenta todo el antecedente teórico expuesto anteriormente, se genera la propuesta de una estrategia de control por retroalimentación no lineal de estados 
obtenida por linealización entrada-salida. Esta ley de control permite regular los AGV en el valor deseado a pesar de errores de modelado (incertidumbres en la cinética de las reacciones) y las restricciones del actuador (valores máximos y mínimos de la tasa de dilución).

La estrategia de control que se propone soluciona el problema del UAFBR bajo estudio. Los resultados simulados indican que puede lograrse un alto desempeño en regulación, como muestra la tabla 5, i.e., bajo tiempo de subida, sobre impulso despreciable y error de estado estacionario insignificante.

A pesar de que un modelo como ADM1, con un alto número de estados permite un buen conocimiento fenomenológico del proceso sin tener que recurrir a costosas corridas experimentales, este tipo de modelos no es recomendable para el diseño de estrategias de control. En este trabajo se demuestra que el uso de modelos reducidos o con pocos estados como AM2 es idóneo para el diseño de leyes de control, considerando que al correrlos sobre modelos complejos nos garantizarán el correcto desempeño de los controladores en la PTAR.

\section{REFERENCIAS}

[1] A. Mottet, I. Ramírez, H. Carrère, S. Déléris, F. Vedrenne, J. Jimenez, y J.P. Steyer, "New fractionation for a better bioaccessibility description of particulate organic matter in a modified ADM1 model", Chemical Engineering Journal, [En línea], vol. 228, pp. 871-881, 2013. DOI: 10.1016/j.cej.2013.05.082

[2] I. Ramírez, "Anaerobic digestion modelling: from one to several bacterial populations", Revista TecnoLógicas , [En línea], vol. 31, pp. 181-201, 2013.

[3] M. Henze, P. Harremos, J. LA Cour Jansen, y E. Arvin, Wastewater Treatment: Biological and Chemical Process, 3. ${ }^{a}$ ed. Springer Science and Business Media, 2001.

[4] D.M. Revelo, N.H. Hurtado, y J.O. Ruiz, "Microbial Fuel Cells (MFCS): a challenge for the removal of organic matter and electricity generation”, Información tecnológica, [En línea], vol. 24, n. ${ }^{\circ}$ 6, 2013. DOI: 10.4067/S0718-07642013000600004

[5] J.P. Steyer, O. Bernard, D.J. Batstone, y I. Angelidaki. "Lessons learnt from 15 years of ICA in anaerobic digesters”, Water Science and Technology, [En línea], vol. 53, n. ${ }^{\circ}$ 4, pp. 25-33, 2006. DOI: $10.2166 /$ wst.2006.107

[6] D.J. Batstone, J. Keller, I. Angelidaki, S. Kalyuzhnyi, S.G. Pavlostathis, A. Rozzi, y V.A. Vavilin. "The IWA anaerobic digestion model no 1 (ADM1)", Water Science and Technology, [En línea], vol. 45, n. ${ }^{\circ}$ 10, pp. 65-73, 2002. DOI: 10.2166/wst.2002.0292

[7] O. Bernard, Z. Hadj-Sadok, D. Dochain, A. Genovesi, y J.P. Steyer, "Dynamical model development and parameter identification for an anaerobic wastewater treatment process", Biotechnology and Bioengineering, [En línea], vol. 75, n. ${ }^{\circ} 4$, pp. 424-438, 2001. DOI: 10.1002/ bit.10036 
[8] S. García-Gen, J.M. Lema, y J. Rodríguez. "ADM1 application to anaerobic co-digestion: generalised implementation of fermentable soluble substrates", Presentado en the $13^{\text {th }}$ world congress on Anaerobic Digestion. Santiago de Compostela, Spain, 2013. DOI: 10.1016/j. biortech.2013.08.063

[9] H.O. Méndez-Acosta, B. Palacios-Ruiz, J.P. Steyer, V. Alcaraz-Gonzáles, E. Latrille, y V. Gonzáles-Alvarez. "Nonlinear approach for the VFA regulation in an anaerobic digester", en $10^{\text {th }}$ International IFAC Symposium on Computer Applications in Biotechnology, 2007. DOI: 10.3182/20070604-3-MX-2914.00015

[10] D. Selisteanu, E. Petre, y V.B. Rasvan, "Sliding mode and adaptative sliding-mod control of a class of nonlinear bioprocesses", International Journal of Adaptive Control and Signal Processing, [En línea], vol. 21, pp. 795-822, 2007. DOI: 10.1002/acs.973

[11] C. Kravaris, y G. Savoglidis, "Tracking the singular arc of a continuous bioreactor using sliding mode control." Journal of the Franklin Institute, vol. 349, n 4, pp. 1583-1601, 2012. DOI: 10.1016/j.jfranklin.2011.06.011

[12] M.R.S. Khan, y R.N.K. Loh, "Nonlinear Control of Bioprocess Using Feedback Linearization, Backstepping, and Luenberger Observers", International Journal of Modern Nonlinear Theory and Application, [En línea], vol. 3, n. ${ }^{\circ}$ 4, pp. 150-162, 2014. DOI: 10.4236/ ijmnta.2014.34017

[13] I. Ramirez, "Integrating Biotechnology and Microbial Ecology in Bioprocesses Control", International Journal of Emerging Technology and Advanced Engineering, vol. 8, n. ${ }^{\circ}$, 2018.

[14] I. Ramirez, "Experimental and modeling investigations of an up-flow anaerobic fixed bed reactor (UAFBR)", Environmental Biotechnology Laboratory, INRA, Narbonne-France, Tech. Rep. LBE-006-69-3, [En línea], p. 160, Abr. 2010. DOI: 10.2166/wst.2008.342

[15] I. Ramirez, y J.P. Steyer, "Modeling microbial diversity in anaerobic digestion”, Water Science and Technology, [En línea], vol. 57, n. ${ }^{\circ}$ 2, pp. 265-270, 2008. DOI: 10.2166/wst.2008.055 